Mundo Agrario vol. 18, nº 38, e053, agosto 2017. ISSN 1515-5994

Universidad Nacional de La Plata.

Facultad de Humanidades y Ciencias de la Educación.

Centro de Historia Argentina y Americana

\title{
Cambios estructurales e institucionales del cooperativismo agrícola entrerriano en la postconvertibilidad (2002-2013). El caso de La Agrícola Regional Cooperativa de Crespo
}

\author{
Structural and institutional changes in agricultural cooperatives of \\ the province of Entre Ríos during the postconvertibilidad stage \\ (2002-2013). The case of La Agrícola Regional Cooperativa of Crespo
}

\section{Ronald Soleno Wilches *}

* Sistema de Investigación, Desarrollo Tecnológico e Innovación -SENNOVA-. Servicio Nacional de Aprendizaje (Sena), Colombia | sronald@sena.edu.co ronaldsoleno@hotmail.com

\section{PALABRAS CLAVE}

Cooperativas agrarias

Cambios estructurales

Mecanismos de adaptación

Contexto competitivo

Siglo XIX

Sistema agroalimentario

Entre Ríos

\section{KEYWORDS}

Agricultural Cooperatives

Structural Changes

Adaptation mechanisms

Competitive environment

Agri-food system

Entre Rios

\section{RESUMEN}

El propósito del trabajo es establecer los principales cambios organizacionales e institucionales desplegados por el cooperativismo agropecuario de la provincia de Entre Ríos (Argentina) para hacer frente a las transformaciones del sistema agroalimentario en la etapa de la postconvertibilidad. La metodología empleada es fundamentalmente cualitativa y se basa en el análisis del caso de La Agrícola Regional Cooperativa de Crespo (LAR). Los hallazgos más importantes permiten observar que las estrategias organizacionales e institucionales adoptadas por LAR le han permitido consolidarse como una "gran empresa cooperativa", cuya compleja estructura (expresada en la multiplicidad de rubros, sucursales, alianzas estratégicas, entre otros) le otorga a la entidad una adecuada capacidad de respuesta frente a las perturbaciones del contexto. El posicionamiento de la misma como organización líder del sistema productivo local, su fuerte arraigo socio-territorial y la naturaleza del entramado de relaciones que se construyen, hacen difícil concebir al territorio sin la presencia de la cooperativa y la existencia de esta última separada de su ámbito de pertenencia.

\section{ABSTRACT}

The purpose of the paper is to establish the main organizational and institutional changes made by the agricultural cooperatives in the province of Entre Rios (Argentina) for facing the agrifood system transformations during the postconvertibilidad stage. The methodology is essentially qualitative and based on the case analysis of La Agrícola Regional Cooperativa of Crespo (LAR). The most important findings show that organizational and institutional strategies adopted by LAR have permitted establish itself as a "Large Cooperative Enterprise," whose complex structure (expressed in the multiplicity of areas, branches, strategic alliances) grants the entity capacity of response to external disturbances. The position of LAR as a leader organization of the local productive system, its strong socio-territorial roots and the nature of the network of relationships built, make it difficult to conceive the territory without the presence of the cooperative and the existence of the cooperative separated from its belonging area. 


\section{Introducción}

Las cooperativas agrícolas son definidas como empresas constituidas y controladas por los productores agropecuarios, cuyo objetivo es proveerles de todos aquellos servicios necesarios para su desarrollo: conservación, transformación, comercialización, provisión de insumos, asistencia técnica, además de fortalecer el poder de negociación, mantener el acceso a mercados competitivos, capitalizar nuevas oportunidades de mercado, mejorar las oportunidades de ingresos y manejar el riesgo. Por ser sus usuarios los dueños no buscan maximizar el beneficio sino minimizar el costo de los servicios (Barton, 2000).

Se debe destacar su importancia como fórmula asociativa y participativa para la generación de sinergias positivas al interior de las zonas rurales, lo que las convierte en protagonistas de la evolución productiva de dichos territorios (Rodríguez, Mozas y Bernal, 2002), dada su importancia en la creación de empleo, la fijación de la población al territorio, su incidencia en el crecimiento empresarial, el desarrollo económico y la mejora del bienestar social de los ciudadanos (Barea, Juliá y Monzón, 1999; Fairbain, 2006). De este modo se alude a la capacidad de este tipo de entidades para estar presente en diversos sectores de la economía, preocupándose no solo por la acción empresarial en su entorno productivo inmediato, sino también por el desarrollo sustentable de las comunidades donde se insertan (Barbero, Gorenstein y Gutiérrez, 2000; De Lisio, 2009).

No obstante, en las últimas décadas y de la mano del proceso de globalización, las cooperativas agrícolas a nivel mundial se han visto sujetas a grandes presiones en distintos niveles (Verheijen \& Heijbroek, 1994; Bijman, Hendrikse \& Veerman, 2000; Anello, Tort y Carballo, 2012). A partir de las presiones, este tipo de organizaciones, poseedoras de una gran fortaleza por su habilidad para generar innovaciones institucionales, han tenido que adaptarse a la cambiante estructura de la producción agrícola, y así suplir sus diversas necesidades económicas (Lang, 1995; King, 1995; King y Ortmann, 2006).

De hecho, las lógicas de acción y arreglos organizacionales de las entidades cooperativas han ido cambiando como respuesta a los cambios antes mencionados y a la consecuente necesidad de capital para afrontar la inserción en los mercados, así como a los riesgos asociados (Nilsson, 1999; Donoso et al., 2003 y 2004; Chaddad y Cook, 2004; Lattuada y Renold, 2004; Lombardo et al., 2009).

Nilsson (1998), Wilson (1999) y O.Connor \& Thompson (2001), argumentan que la búsqueda por ganar una mayor competitividad y sobrellevar las limitaciones del modelo tradicional ha traído como resultado que las cooperativas se hayan visto obligadas a romper con prácticas pasadas y a examinar nuevos enfoques, nuevas políticas, nuevas estructuras y por ende nuevos modelos (Barton, 1989; Cook, 1995; Nilsson, 1998).

Estas innovaciones organizativas o "modelos cooperativos no tradicionales" surgen en principio en los Estados Unidos, aunque se han ido extendiendo poco a poco a otras partes del mundo. Los trabajos de Chaddad y Cook (2004) y de Von Pischke y Rouse (2004), identifican tres modelos que suponen la eliminación de ciertas restricciones frente al modelo tradicional y en los que se produce un aumento del incentivo a invertir por parte de sus socios, las cooperativas de inversión proporcional, las cooperativas de socios inversores y las cooperativas de nueva generación.

Además de estos modelos no tradicionales, Cook y Chaddad (2004), y Basáñes (1999 y 2004), resaltan la existencia de otras estrategias tendientes a incentivar la inversión, y por ende a dar lugar a una mayor capitalización en las cooperativas agrícolas. Dentro de las mismas se destacan los programas de capitalización por objetivos, las sociedades instrumentales y las cooperativas que cotizan en bolsa.

En una línea de análisis distinta, Lattuada y Renold (2004), al abordar el proceso de transformación institucional del cooperativismo agropecuario, se refieren a formas de organización que se diferencian sustancialmente unas de otras, permitiendo en una etapa intermedia la convivencia de normas y prácticas contradictorias y llegando a un punto de inflexión (la etapa de mutación) que implicaría dos alternativas de desarrollo institucional opuestas: el retorno a una mayor coherencia entre las prácticas y normas originales, o a una drástica modificación de los principios originarios que da lugar a un funcionamiento de la organización más como una empresa administradora de capital que como una cooperativa. 
En el primer caso, serán cooperativas relativamente pequeñas, locales, con alta participación y compromiso de sus socios, con escasa burocratización interna, con funciones que abarcan más allá de la relación económica comercial, incluyendo ayuda solidaria y gestiones múltiples -legales, financieras, gremiales, etc. - en beneficio de sus socios, y un nivel de retribución claro, inmediato y transparente de parte de los beneficios que pueda obtener la cooperativa (Lattuada y Renold, 2004).

El segundo caso implica "grandes empresas cooperativas" con expansión territorial, sin límites de sus negocios, asociaciones y control de empresas de capital, operación con socios y no socios, gran desarrollo de su administración a partir de un cuerpo profesional ajeno a los socios, prioridad de la eficiencia económica y administrativa de la empresa. Los factores de mayor compromiso entre los asociados y las cooperativas ya no se basan en una relación de tipo asociado/propietario o asociado/cliente, sino en la subordinación del productor a la empresa, debido a las exigencias de frecuencia, cantidad, y calidad en la producción que exige la incorporación al sistema agroalimentario (Lattuada y Renold, 2004).

\section{Antecedentes y contexto argentino}

El surgimiento del cooperativismo agrario en Argentina se remonta a las últimas décadas del siglo XIX y está relacionado de manera directa con el proceso inmigratorio europeo registrado en la época, propiciado por las políticas económicas imperantes en ese entonces ${ }^{\underline{1}}$ (Cracogna, 1968; Caletti, 1983; Salminis, 2003 y 2010). La primera experiencia de la que se tiene registro es la Sociedad Cooperativa de Seguros Agrícolas y Anexos Limitada "El Progreso Agrícola", creada en 1898 por un grupo de colonos franceses de Pigüé, provincia de Buenos Aires.

Posteriormente, en Entre Ríos surgieron varias iniciativas de los inmigrantes israelitas y alemanes asentados en la zona, viéndose materializadas por primera vez en el año 1900 cuando se constituyó la cooperativa La Agrícola Israelita, que siete años después pasaría a denominarse Sociedad Agrícola Lucienville; luego, en 1904, se

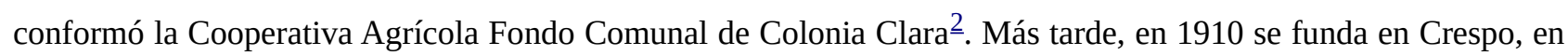
la misma provincia, la Sociedad Anónima Cooperativa Limitada La Agrícola Regional $\underline{3}$ (López, 2008). Además se destaca la conformación en 1913 de la primera cooperativa de segundo grado, La Confederación Entrerriana de Cooperativas 4 (Salminis, 2010), que tuvo un muy breve lapso de existencia y fue refundada en 1930 con el actual nombre de Federación Entrerriana de Cooperativas (Mateo, 2011).

Avanzando en el tiempo es posible observar la manera en que el cooperativismo agrario argentino tiene su mayor auge entre los años 1930 y 1960. En este periodo, específicamente en el año 1956, surge la Confederación Intercooperativa Agropecuaria Limitada (CONINAGRO), constituyéndose en la primera cooperativa de tercer grado del país (Ressel y Silva, 2008). El proceso de crecimiento del sector estuvo avalado por el número de cooperativas fundadas en dicho periodo, la integración institucional y empresarial del sistema, la expansión del movimiento en el sector agroindustrial y comercial, y la definición de un marco jurídico especifico (Gorenstein et al., 2001; Salminis, 2003 y 2010).

No obstante ello, a partir de la reforma financiera de 1977, comenzaron las manifestaciones de crisis del sistema cooperativo (Lattuada, 2006). Según lo expresan Gorenstein et al. (2000 y 2001), en el ciclo de mortandad de las cooperativas agropecuarias pampeanas pueden diferenciarse dos etapas. La primera se produjo durante el inicio de la década del 80 bajo los efectos directos de la reforma financiera, cuando las cooperativas empezaron a tener dificultades de cobranza, llegando muchas de ellas a crisis de insolvencia. La segunda se dio durante la década de los 90, cuando la situación económico-financiera de las cooperativas agrarias, sobrevivientes a los severos cambios de la década anterior, condicionó, en buena medida, sus posibilidades empresariales en un contexto macroeconómico y sectorial caracterizado por la aplicación de un modelo de apertura y desregulación económica, que a su vez incluía la convertibilidad del peso, la disminución de aranceles de importación, la eliminación de organismos reguladores del comercio y la producción agropecuaria, las privatizaciones y el fomento de la 
inversión extranjera (Obschatko et al., 2011).

Por su parte, otros autores afirman que durante este periodo se registró un proceso de transformaciones institucionales en las cooperativas agropecuarias de la región pampeana, lo que determinó una serie de cambios organizativos y de acción, los que a su vez ocasionaron tensiones entre los principios doctrinarios y el accionar cotidiano (Romero et al., 2006). Las modificaciones involucraron tanto a la relación de los productores con sus cooperativas como al vínculo entre las cooperativas y los restantes eslabones de la cadena (Lombardo et al., 2009).

Durante la etapa posterior a la crisis del 2001 aparece un nuevo marco de política macroeconómica, caracterizado por una fuerte expansión del mercado interno, la sustitución de las importaciones, la fuerte dinámica exportadora y la expansión de la inversión. De este modo, además de la política cambiaria caracterizada por la devaluación de la moneda y de las políticas de desarrollo del sector industrial, se destaca la inclusión de mecanismos de acceso al crédito, asistencia técnica, capacitación y desarrollo por parte de las pequeñas y medianas empresas (Fulkas, 2009). En este escenario, según Obschatko et al. (2011), la política agropecuaria ha prestado especial interés a la situación de los pequeños productores y agricultores familiares y a la promoción del asociativismo, fortaleciendo programas anteriores y creando nuevos. Como resultado de lo anterior, se dio lugar a la conformación de un gran número de asociaciones de productores agropecuarios, en paralelo a la creación de programas de fomento para la Agricultura Familiar.

Entre los estudios existentes sobre el cooperativismo agrario en la etapa de postconvertibilidad, es preciso destacar el trabajo de Ressel y Silva (2008), en el cual se plantea que la devaluación de la moneda permitió que el agro recibiera una mejora sustantiva en el tipo de cambio, y que se viera beneficiado por la revalorización de los precios internacionales. Esto, sumado a otros factores, propició que los pequeños productores agropecuarios se unieran conformando nuevas experiencias asociativas, entre ellas las de tipo cooperativo $\underline{5}$. No obstante, también sostienen que persisten obstáculos para los pequeños y medianos productores agropecuarios, relacionados con la necesidad de reconvertir sus sistemas productivos para poder mantenerse en el contexto del modelo económico imperante (Ressel y Silva, 2008).

Lattuada, et al. (2011), en su trabajo más reciente, abordan el cooperativismo agropecuario argentino desde la perspectiva del capital social -entendido como los lazos y redes de cooperación entre actores individuales y entre organizaciones que contribuyen al desarrollo de los territorios-. En el mismo se abordan tres casos de cooperativas: FeCoVita, Sancor y Agricultores Federados Argentinos, vinculando la literatura especializada en capital social con los aspectos centrales del análisis morfológico de dichas organizaciones. En una línea de análisis similar, Olivera (2008) sostiene que la noción de economía social sustenta cultural, ideológica y políticamente, a un conjunto de iniciativas asociativas que propician el desarrollo endógeno y la gestión participativa (como es el caso de las cooperativas agrarias), y que apuntan a incrementar la capacidad de pequeños y medianos productores agropecuarios de hacer frente a políticas de apertura y desregulación, concentradoras de la estructura social agraria.

A los antecedentes citados, sumamos los planteos de López (2008), según los cuales los análisis respecto al cooperativismo agrario argentino en su mayoría se han centrado en la región pampeana, dejando de lado muchas veces a las experiencias de este tipo asentadas en otras provincias, entre ellas la de Entre Ríos. Según lo expresa López (2008), los trabajos existentes profundizan en las razones del ocaso de las entidades pioneras del sector, indicando entre otras cosas, que el crecimiento institucional (a veces demasiado rápido) encontró a las cooperativas faltas de una dirigencia que supiera sortear la situación y organizar debidamente el avance de las entidades, lo que inevitablemente llevó al ocaso del modelo pionero. Para la autora: “dicho análisis no cubre la experiencia entrerriana, que escapa en todos sus aspectos a los parámetros del modelo en cuestión, tomando las características de un modelo propio, de notable crecimiento institucional...” (López, 2008, p. 369).

En ese sentido, el objetivo del presente trabajo es el de establecer las principales estrategias y/o cambios organizacionales e institucionales a través de los cuales el cooperativismo agrario de la provincia en mención ha 
logrado responder a la necesidad de mantenerse y reconvertirse, y la forma en que dichos cambios influyen en las relaciones establecidas con asociados y la población en general, en la generación de iniciativas de desarrollo local y la formación de capital social. Para tal fin ponemos foco en La Agrícola Regional Cooperativa de Crespo, una entidad de larga data (fundada en 1910 por colonos inmigrantes alemanes del Volga, italianos y algunos criollos) alrededor de la cual giran la mayor parte de las actividades productivas y la prestación de servicios al interior del área estudiada. El estudio de la misma resulta interesante en función de aspectos como su dimensión organizacional, la multiplicidad de unidades de negocio, el número de asociados y empleados directos, su capacidad de establecer alianzas con otras cooperativas y empresas de capital, y la trayectoria e importancia a nivel local y provincial.

\section{Metodología}

La metodología es del tipo cualitativo, basada en un análisis exploratorio que pone énfasis en el caso seleccionado. Para la realización del análisis de caso se recopiló información secundaria relacionada con la temática en particular (artículos científicos, informes provinciales, páginas web, etc.) y referida a la cooperativa seleccionada (Estatutos, libros, periódicos, balance social, etc.). Además se recopiló información primaria proveniente de ocho entrevistas en profundidad realizadas en el año 2013 a miembros del consejo directivo, la gerencia e informantes calificados seleccionados en función del rol que desempeñan dentro del sistema productivo (gobierno local, INTA, entidades educativas, etc.). Además se realizaron entrevistas semiestructuradas a productores incluidos en las iniciativas innovadoras (horticultura y producción de especias) de la cooperativa [N de E.: Suprimido], a los que se les indagó sobre la importancia de la presencia de la entidad en la zona.

Las entrevistas a miembros del consejo directivo y la gerencia giraron en torno a los orígenes del emprendimiento, los objetivos perseguidos, las etapas atravesadas, modelos de organización y gestión internos, naturaleza del vínculo establecido con sus asociados, prácticas productivas y comerciales utilizadas, entre otros aspectos. Las entrevistas a informantes calificados estuvieron orientadas principalmente a indagar sobre la percepción de los actores respecto a la importancia de la entidad para el desarrollo socioeconómico local.

\section{Caracterización del área geográfica de estudio}

El área estudiada comprende la zona de la ciudad de Crespo -ubicada en el departamento de Paraná, en los límites con los departamentos de Diamante y Nogoyá (ver figura 1) -, y las aldeas cercanas como Estación General Racedo, Estación Camps, Villa Gdor. Etchevehere, Aldeas Eigenfeld, Santa Rosa, San Rafael, San Juan, San Miguel y Colonias Reffino y Merou, contando con una superficie total de 57.500 ha, de las cuales 51.000 ha (cultivos y pasturas) se encuentran en producción (INTA EEA Crespo, 2013).

En la zona se registran 295 unidades productivas -dedicadas principalmente a la avicultura, porcicultura, ganadería y cultivos alternativos- (INTA Crespo, 2013), cuyo tamaño promedio es de 170 ha. Además se observa una estructura productiva diversificada y sustentada principalmente en pequeños y medianos productores, donde la mano de obra es ampliamente demandada para realizar trabajos intensivos.

$\mathrm{Al}$ interior del sistema se destaca la presencia de actores sociales de distinta índole, que en su conjunto configuran el entramado de relaciones económico-productivas que dinamizan los subsistemas y encadenamientos subyacentes. Dentro de ellos se destaca la presencia de la Agrícola Regional Cooperativa Ltda., una entidad alrededor de la cual giran la mayor parte de las actividades productivas y la prestación de servicios de todo tipo, a pesar de la existencia de otras empresas locales con proyección regional y nacional (especialmente en la producción avícola). 


\section{Figura 1. Localización geográfica de Crespo, Entre Ríos}

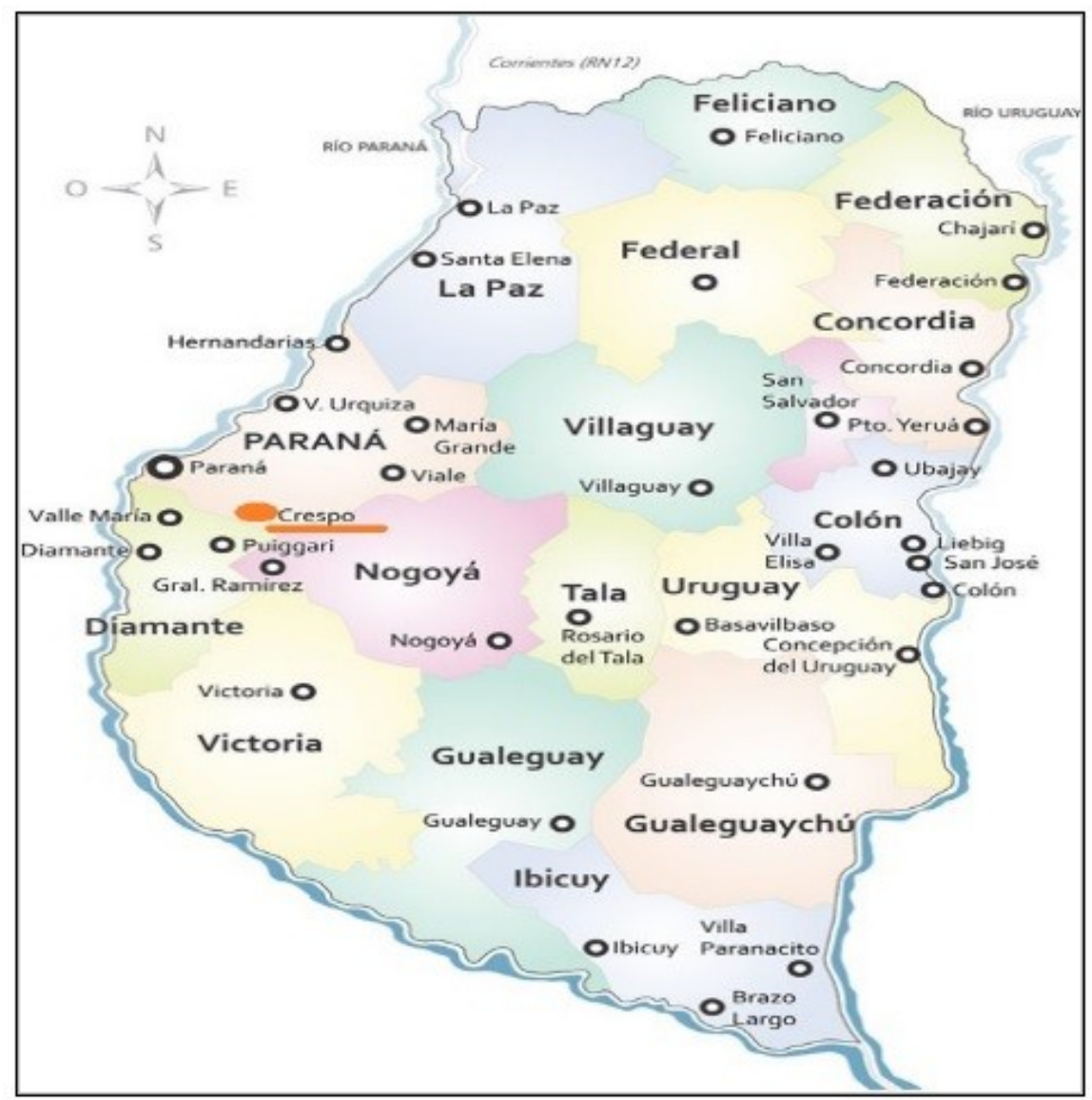

Fuente: www.fca.uner.com.ar

\section{El caso de La Agrícola Regional Cooperativa Ltda. (LAR)}

Fue fundada el 29 de abril de 1910 como una iniciativa de los colonos de la zona de Crespo, encabezados por Alejandro Mohor $\underline{\underline{6}}$ y Otto Sagemüller ${ }^{7}$, que se planteaban en su momento la necesidad de conformar una sociedad de productores que les permitiera comercializar la producción granaria en forma conjunta, buscando hacer frente a las grandes firmas acopiadoras con presencia en la zona, y a la vez asegurarse la provisión de los insumos necesarios para el desarrollo de la actividad agrícola a un menor precio (semillas, herramientas, lubricantes, veneno para las hormigas, bolsas e hilos). De este modo 193 productores, en su mayoría inmigrantes alemanes del Volga, italianos y algunos criollos ${ }^{\underline{8}}$, se propusieron defender su producción aplicando el sistema cooperativo de sus países de origen (López, 2008; Balance social LAR, 2012-2013).

Pero a pesar de priorizar los valores cooperativos, las leyes imperantes en la época no contemplaban adecuadamente a esa nueva forma asociativa $\stackrel{9}{\text {; }}$ por tal razón la entidad tomaría en principio la denominación de Sociedad Anónima Cooperativa Limitada La Agrícola Regional, firmando en ese entonces una prorroga de 30 años de duración (el contrato social expiraría el 29 de abril de 1940). Es por esto que, muy a pesar de la promulgación de la primera ley de cooperativas en el país (Ley № 11388) en 1926, sólo hasta el año 1939 se trató nuevamente el tema de la denominación de la sociedad. No obstante, y teniendo en cuenta que algunas de las disposiciones de la ley no presentaban las ventajas esperadas por los asociados, se decidió prorrogar el contrato social como estaba por 15 años más (hasta el 29 de abril de 1955). La adopción de la figura cooperativa se concretó de manera formal en el año 1952, cuando por decisión tomada en asamblea general extraordinaria quedó registrada como tal ante la Dirección Nacional de Cooperativas del Ministerio de Industria y Comercio (Wagner, 
2011).

El propósito de la cooperativa es adquirir o producir artículos de consumo, de uso personal y del hogar para ser distribuidos entre los asociados, así como comercializar cereales y demás productos de origen vegetal y/o animal en el mercado interno o externo. Para tal caso, estima pertinente la provisión de maquinaria, repuestos, enseres, bolsas, hilos, semillas y todos los insumos necesarios. Además se plantea la construcción de fábricas y/o establecimientos adecuados para la manipulación, conservación, industrialización y distribución de la producción de sus asociados. En concordancia con esto, se prevé el otorgamiento de créditos y adelantos en dinero, a cuenta de la producción entregada, para facilitar las operaciones inherentes.

Otras actividades contempladas se relacionan con la adquisición y/o arriendo de campos y la provisión de energía eléctrica a los productores vinculados, el prestar asesoría técnica y jurídica y fomentar el espíritu de previsión y economía. De la misma manera, se busca el mejoramiento de la industria agraria y la defensa de los intereses de los asociados, auspiciar y organizar consorcios camineros, crear conciencia cooperativa, propender por el abaratamiento de los costos de producción y la simplificación de la comercialización, prestar servicios, obras o actividades tendientes a promover el bienestar de los asociados y fomentar la educación cooperativa (Estatuto LAR, 1952).

De todo esto se puede inferir que, a pesar de que cobra relevancia la lógica empresarial, también se contempla en el discurso la defensa de los intereses de los asociados y el cumplimiento de los principios propios de la actividad cooperativa. Más allá de estas consideraciones, que nos dan una idea respecto a las pautas y/o los objetivos trazados por la cooperativa desde sus inicios, resulta interesante, de acuerdo con el objetivo del trabajo, poder establecer la forma en que la entidad ha enfrentado los cambios que el sistema agroalimentario argentino ha experimentado durante las últimas dos décadas (1990-2013).

Para ello, se hace imperativo mencionar antes que, durante las primeras dos décadas que siguieron a su conformación, LAR enfocó sus esfuerzos en el acompañamiento de los socios productores, facilitándoles el acceso a insumos, semillas y seguros agrícolas $\frac{10}{}$, así como la obtención de precios favorables por la venta de la producción. Durante este periodo se fundó la sección de materiales para la construcción y el almacén de ramos generales (maquinaria, yerba, café, azúcar, arroz, jabón, kerosene, etc.). Se dio inicio a un proceso de crecimiento progresivo, acompañado por la apertura de sucursales en localidades vecinas (Tabosi y Hernández). Asimismo, se realizaron inversiones para el acopio de cereales (incluyendo silos y sistemas de secado y pesaje), fábrica de alimentos balanceados (NutriLAR), electrificación rural, comercialización de leche (convenio con la cooperativa de segundo grado Sancor), cría de cerdos, alianzas con Seguros La Segunda y ACA Salud. También se conformó el grupo de jóvenes cooperativistas, se dio inicio a la sistematización informática, se realizaron múltiples procesos de ampliación y remodelación del supermercado central, y se incursionó en la provisión de lubricantes y gasoil (YPF).

\subsection{LAR durante la convertibilidad (1990 - 2001)}

En la década del 90, en un contexto caracterizado por la retracción del Estado en relación a las actividades productivas, la desregulación y apertura de la economía, la convertibilidad, las privatizaciones del sector público, el endeudamiento externo, mayor presión fiscal y la flexibilización laboral, desde la dirección se empezaron a percibir problemas asociados a la gestión de la cooperativa. Ello dio lugar a un proceso de reestructuración administrativo-organizacional que, entre otros aspectos, incluyó la conformación de un equipo de trabajo profesional y la capacitación del personal encargado de las labores de gestión: "Se dieron cuenta que la falla estaba en la gestión, que era una empresa que se gestionaba casi como al principio, y ya venía todo ese quiebre en el management...entonces empezaron a capacitarse” (Entrevista a informante calificado de Crespo, 2013).

Por otra parte, las condiciones del entorno impactaron de manera negativa sobre la actividad lechera generando el retiro de gran parte de los productores. No obstante, aquellos con mayor capacidad económico-productiva realizaron inversiones en sistemas de frío y mejoraron sus instalaciones, logrando el acompañamiento de la 
cooperativa a través de la renovación del convenio con Sancor (en la actualidad permanecen 15 tamberos que trabajan de manera exclusiva).

En concordancia, y a pesar del contexto desfavorable de ese entonces, la cooperativa continuó con la estrategia de crecimiento, viéndose materializada en la construcción del denominado Complejo Agronómico de Crespo con una capacidad de acopio de 39.500 t de cereales. Al tiempo se trasladó la fábrica de chacinados (actividad desarrollada desde los inicios de LAR en las instalaciones de la casa central) a un predio contiguo, incluyendo cámaras frigoríficas y montaje de maquinarias entre otras cosas. A la par se llevó a cabo la renovación y ampliación del supermercado central. También en esta década se concretó la compra de acciones del Molino Harinero Ramírez S.A. $\underline{11}$ en conjunto y en partes iguales con ACA y la Cooperativa Ganadera Gral. Ramírez. Paralelamente se creó la firma Tecnovo por parte de la Sociedad Anónima de Productores Avícolas, entre los que se encuentra LAR (como socio accionario). Se inauguró la estación de servicios YPF de Crespo y se adquirió la agencia representante de seguros la Segunda en la ciudad de Paraná. LAR además logró ser habilitada como agencia de viajes y turismo bajo la designación Coovaeco Turismo en Cooperación. Empezó la producción de ganado de engorde en corral (feed-lot) y se refaccionaron las instalaciones de la ferretería, inaugurando al mismo tiempo un autoservicio. Se inauguró un sistema de distribución diesel (con entrega de combustible en el establecimiento del asociado, a través de camiones cisterna).

Vale destacar que durante este periodo desde la cooperativa se avizoraron los posibles efectos de la crisis sobre los pequeños productores familiares, por lo que se empezaron a generar iniciativas tendientes a asegurar su persistencia. De este modo surgió el Grupo Hortícola LAR, conformado inicialmente por 10 familias ubicadas en las aldeas aledañas (Gutman, 2005; Balance Social LAR, 2012; Soleno y Craviotti, 2014).

Hasta este punto se puede observar la forma en que LAR, en función de los objetivos planteados, dio continuidad a un proceso de crecimiento sostenido, caracterizado por la apertura de nuevas unidades de negocios, inclusión de servicios de distinta índole, apertura de nuevas sucursales, participación accionaria en empresas de capital, entre otros aspectos, además de la adopción de procesos de mejora a nivel organizacional. Todo ello permitió a la entidad poder afrontar situaciones adversas sin que ello afectara de manera notoria sus principios cooperativos. De hecho, puso en marcha proyectos orientados a asegurar la permanencia de la producción familiar y para fomentar la educación cooperativa.

Asimismo, frente a la agudización de la crisis en el año 2001, gestionó otras iniciativas para ayudar a paliar los efectos negativos. Como ejemplo se puede citar el proyecto "Comamos con soja”, en paralelo con el programa “Soja Solidaria” $\underline{12}$. Otra iniciativa interesante en términos de responsabilidad social y que ayudó a posicionar a la cooperativa durante la crisis fue la aceptación de los denominados bonos federales $\underline{13}$ a valor nominal:

Los productores tenían deudas en dólares y la provincia estaba complicada, había una pseudomoneda que eran los federales, así que nosotros acompañamos muchísimo a nuestros consumidores, recibíamos los federales a su valor nominal y en la competencia lo recibían al 50\% porque estaba totalmente devaluado... (Entrevista a Gerente de LAR, 2013).

Esto fue clave para el crecimiento en los distintos rubros y particularmente los relacionados con los servicios (electrificación) y consumo (supermercados, estaciones de servicio), ya que la gente podía pagarlos con dicha moneda. De esta manera, y según lo manifiestan los informantes calificados entrevistados, la cooperativa logró afianzar el vínculo con sus asociados y con la comunidad en su conjunto. Todo esto la ubicaría como una organización institucional en mutación, bastante afín al subtipo mutualista (Lattuada y Renold, 2004), dada su evidente preocupación por revalorizar las lógicas que sustentan la actividad cooperativista. 


\subsection{La etapa postconvertibilidad (2002 - 2013)}

A partir del año 2002 la cooperativa siguió su proceso de capitalización y expansión territorial, facilitado por un contexto de reposicionamiento de las exportaciones que le era favorable al productor agropecuario. De esta manera, no sólo se realizaron inversiones y adquisiciones, sino que surgieron nuevas iniciativas para el apoyo a la pequeña y mediana producción (con posibilidades de ser excluida del sistema), tal es el caso del grupo de hierbas aromáticas y condimentarias formado en el año 2002.

Por otra parte, en 2003 se inauguraron en Nogoyá dos supermercados minoristas, Sur (800 m²) e Italia (300 m²):

El dueño de dos supermercados vino a la gerencia y les dijo que por favor, que él estaba ya aniquilado, pero lo que no quería era desaparecer, sobre todo por sus 20 empleados, entonces se los vendía a largo plazo, pero que tomaran los empleados...por esa función social se instaló [LAR] en Nogoyá (Entrevista a informante calificado Crespo, 2013).

Al tiempo se abrió la sucursal Alcaraz (oficina de compra de cereales y oleaginosas, así como de servicios e insumos), que desde 2006 cuenta con una planta de acopio de cereales con una capacidad total de 10.100 t. También se amplió la capacidad de acopio de la sucursal Valle María (Departamento de Diamante) que actualmente cuenta con una capacidad de $4.600 \mathrm{t}$.

En 2003 también se registra la construcción de un salón de exposición y ventas para el corralón de materiales, que sería ampliado en 2004, año en que se abrió la sucursal María Grande y se firmó un acuerdo con el grupo asegurador La Segunda para abrir 5 nuevas sucursales en la ciudad de Paraná. Durante este periodo LAR dejó de ser agente mayorista y se convirtió en distribuidor de combustibles y lubricantes YPF (instaló una plataforma para la carga de combustible en los camiones y una agencia en Paraná). También se concretó la renovación de la maquinaria del Molino Harinero de Ramírez, se puso en funcionamiento el centro de distribución y supermercado mayorista de Crespo, y se inauguró el actual "paseo de compras” que implicó la remodelación del supermercado de la casa central y su posterior ampliación en 2008. Además se inauguró una planta de hormigón elaborado en el parque industrial de Crespo. Otra iniciativa surgida tiene que ver con un proyecto para la producción y comercialización de nuez pecán, enfocado en productores capitalizados interesados en diversificar la producción al interior de sus explotaciones $\underline{14}$.

El final de la última década encuentra a la cooperativa con una gran estructura de negocios, que trasciende el ámbito local, posicionada en otras localidades paranaenses y de los vecinos departamentos de Diamante y Nogoyá. Cuenta con cerca de $4750 \underline{15}$ asociados y 600 empleados directos, centrando sus mayores esfuerzos en el despliegue de estrategias de innovación organizacional, tecnológica, productiva y comercial, de manera que se optimicen los procesos y se mejore la calidad de los servicios prestados tanto a los asociados como a los clientes externos. No obstante, como organización económica no ha sido ajena a las repercusiones de la crisis global registrada en el año 2008, que tuvo su impacto en el sistema agroalimentario internacional y subsecuentemente en el ámbito local.

Además de ello, las retenciones sobre las exportaciones como elemento de política económica $\frac{16}{}$ a nivel país, ha supuesto un escenario complejo para la producción agropecuaria; sin embargo, su gran estructura y diversificación económico-productiva, le confieren a la cooperativa una gran flexibilidad y margen de maniobra frente a las perturbaciones y/o los efectos adversos del contexto.

Pero más allá de los aspectos relacionados con su estructura productiva, cabe mencionar que LAR ha logrado mantener desde sus inicios un esquema organizativo flexible en el que se separa la dirección de la gerencia. La dirección está a cargo de un CDA compuesto por nueve miembros titulares (presidente, vicepresidente, secretario, prosecretario, tesorero, protesorero y tres vocales) y tres suplentes elegidos en asamblea $\frac{17}{}$, al igual que un síndico titular y un suplente para cada ejercicio. Por su parte, la gerencia $\frac{18}{}$ (en quien recaen las responsabilidades en la toma de decisiones estratégicas que tienen que ver con el posicionamiento de la cooperativa en el ámbito social, 
económico, productivo y comercial) está a cargo de personal profesionalizado, al igual que el personal de apoyo (finanzas, relaciones institucionales, contabilidad, recursos humanos, sistemas impositivos, sistemas informáticos, marketing, auditoria interna, créditos y cobranzas), factor que cobró importancia a partir la década del 90 (ver figura 2).

\section{Figura 2. Organigrama de la Agrícola Regional Cooperativa}

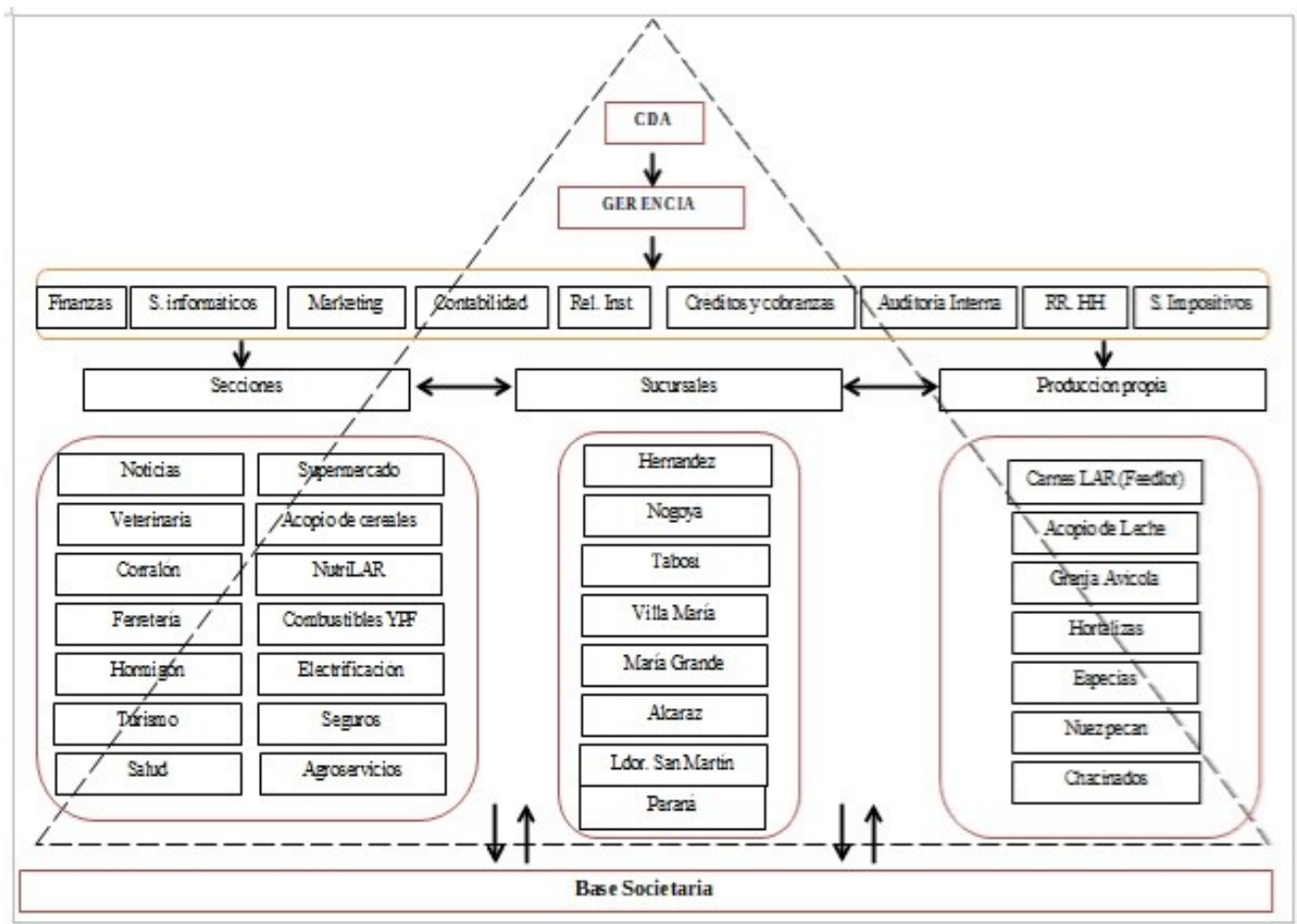

Fuente: Elaboración propia.

Las asambleas fungen como las estancias máximas para la toma de decisiones por parte de los asociados; las mismas pueden ser ordinarias $\frac{19}{}$, de carácter anual, y extraordinarias. Sin embargo, un dato que se debe considerar es el bajo porcentaje de asistencia a las mismas (inferior al 3\% de la base societaria) (Balance social LAR, 2013); por esta razón desde la Presidencia del CDA y la Gerencia se ha adoptado un esquema en el cual se realizan reuniones periódicas en las distintas localidades en las que se tiene presencia con el objetivo de informar las novedades y al tiempo conocer las problemáticas comunes:

A las asambleas viene te diría el 10\% de los socios, más no viene y a veces menos...hacemos reuniones en las sucursales...donde va parte del CDA y muchos de los integrantes del equipo estratégico, vamos y hacemos reuniones donde les hablamos de empresas de familia, hablamos de distintas producciones, $\mathrm{y}$ también nos sentamos a escucharlos... (Entrevista a Gerente de LAR, 2013).

Esto pone en evidencia una de las dificultades que, según la bibliografía consultada, enfrenta este tipo de organizaciones como producto del proceso creciente y sostenido de capitalización que complejiza su estructura, afectando la relación que se establece entre la entidad y sus asociados. Estos últimos ven limitadas sus posibilidades para influir en las decisiones que toma la organización. Pese a ello, es destacable en este caso la existencia de un mecanismo que permite a la entidad un acercamiento a las problemáticas de los productores que nuclea.

En la actualidad la cooperativa opera con asociados y no asociados. Generalmente las actividades de producción propia requieren que el productor se encuentre vinculado a la entidad, no siendo estrictamente necesario para el consumo de bienes y servicios (supermercado, ferretería, corralón de materiales, entre otros): 
Quedó que atiende a asociados y clientes, es decir, puede haber quien quiera proveerse aquí en el supermercado, el que necesite energía eléctrica en el campo y no tenga interés de asociarse. Ese es un cliente, el excedente que genera se capitaliza a favor de la cooperativa mientras que el asociado el excedente que genera por la operatoria se le capitaliza en su cuenta de capital (Entrevista a Responsable de las Relaciones Institucionales de LAR, 2013).

Tal como se desprende de la cita anterior, no se distribuyen excedentes al final de cada ejercicio, más allá del beneficio obtenido por cada asociado en función de su actividad cooperativizada, después de los gastos y/o deducciones a que haya lugar por parte de la cooperativa $\underline{20}$. De este modo, existe un sistema en el cual al productor se le deposita en cuenta corriente el pago de la producción entregada y los excedentes provenientes de las demás actividades se destinan al crecimiento/capitalización de la entidad: Es algo como que ya está asumido por parte de los asociados que alguna vez entendieron que si se llevan los excedentes no se puede reinvertir (Entrevista a Responsable de las Relaciones Institucionales de LAR, 2013).

Una situación emergente que vale la pena destacar, es que no se contempla la obligatoriedad de entrega del total de la producción: El asociado no está obligado a entregar toda su producción” (Entrevista a Responsable Relaciones Institucionales, 2014). Sin embargo, tal y como se lee en el Estatuto se habla de una obligatoriedad de entrega de la producción que ésta pueda distribuir y/o industrializar, con lo cual los volúmenes a entregar a la cooperativa dependen directamente de las condiciones del mercado. Sin embargo la falta de fidelidad o compromiso puede resultar problemático en temporadas de alta demanda y escasa oferta de un producto agrícola determinado, más aún si se tiene en cuenta la inexistencia de penalidades y que muchos productores se encuentran a la vez vinculados a otras cooperativas de la zona $\underline{21}$.

Esto guarda estrecha relación con la distribución geográfica de los campos, ya que en función de dicha variable, los productores optan por asociarse también a aquellas entidades cercanas en las que puedan entregar la producción con el mínimo costo posible:

Acá tenemos la cooperativa de Diamante que está a $30 \mathrm{Km}$, está la cooperativa de Aranguren que está a 20 Km, está la Ganadera de Ramírez que está a 30 Km, así hay 5 o 6 cooperativas, está ACA, entonces hay productores que son socios de varias cooperativas porque viven acá en Crespo pero tienen campo cerca de Diamante, entonces para no traerlo acá que les parece mucho flete, se hacen socios y entregan allá (Entrevista a Gerente de LAR, 2013).

Desde la cooperativa se apela a la necesidad de adaptarse a las condiciones del contexto, ofreciéndole al productor una mayor flexibilidad. Pero ello requiere a su vez del despliegue de estrategias a través de las cuales se logre un mayor grado de compromiso y sentido de pertenencia hacía a la entidad: El asociado quiere tener más libertad...se llegó a la conclusión de que el asociado tiene que ser libre y la cooperativa tiene que tentarlo en todo caso con mejores condiciones (Entrevista Informante Calificado de LAR, 2014). En ese sentido resulta importante el ofrecer a los productores un sistema de pago estable y confiable, el acceso a insumos y servicios a precios favorables, así como la generación de actividades que agreguen valor a la producción agropecuaria.

Para ello se considera necesaria la integración con otras entidades cooperativas y organizaciones de distinta índole (ver figura 3): Estamos con esa cuestión de seguir agregando valor y seguir fortaleciendo la presencia de la cooperativa (Entrevista a Gerente de LAR, 2013. Esto lo demuestra el establecimiento de alianzas y contratos con la Cooperativa Obrera de Bahía Blanca (Marca "Primer Precio"), la Cooperativa Arrocera de Villaguay, la Cooperativa de Carniceros de Paraná (faena de ganado vacuno engordado por LAR), y con varias cooperativas de servicios públicos (Cooperativa de Seguí, Cooperativa eléctrica de Gálvez, Santa Fe), de seguros y servicios (salud, turismo, etc.). Además, el entramado de relaciones con entidades de segundo y tercer grado como la Asociación de Cooperativas Argentinas (ACA), la Federación Entrerriana de Cooperativas (FEDECO), la Federación Argentina de Cooperativas Eléctricas (FACE), Sancor y la Confederación Intercooperativa Agropecuaria (CONINAGRO). A esto se suman las alianzas estratégicas (y participación accionaria) con 
empresas de capital como Tecnovo, el Molino Harinero de Ramírez, Alimentos Segemuller, entre otras.

Figura 3. Entramado de relaciones de La Agrícola Regional Cooperativa

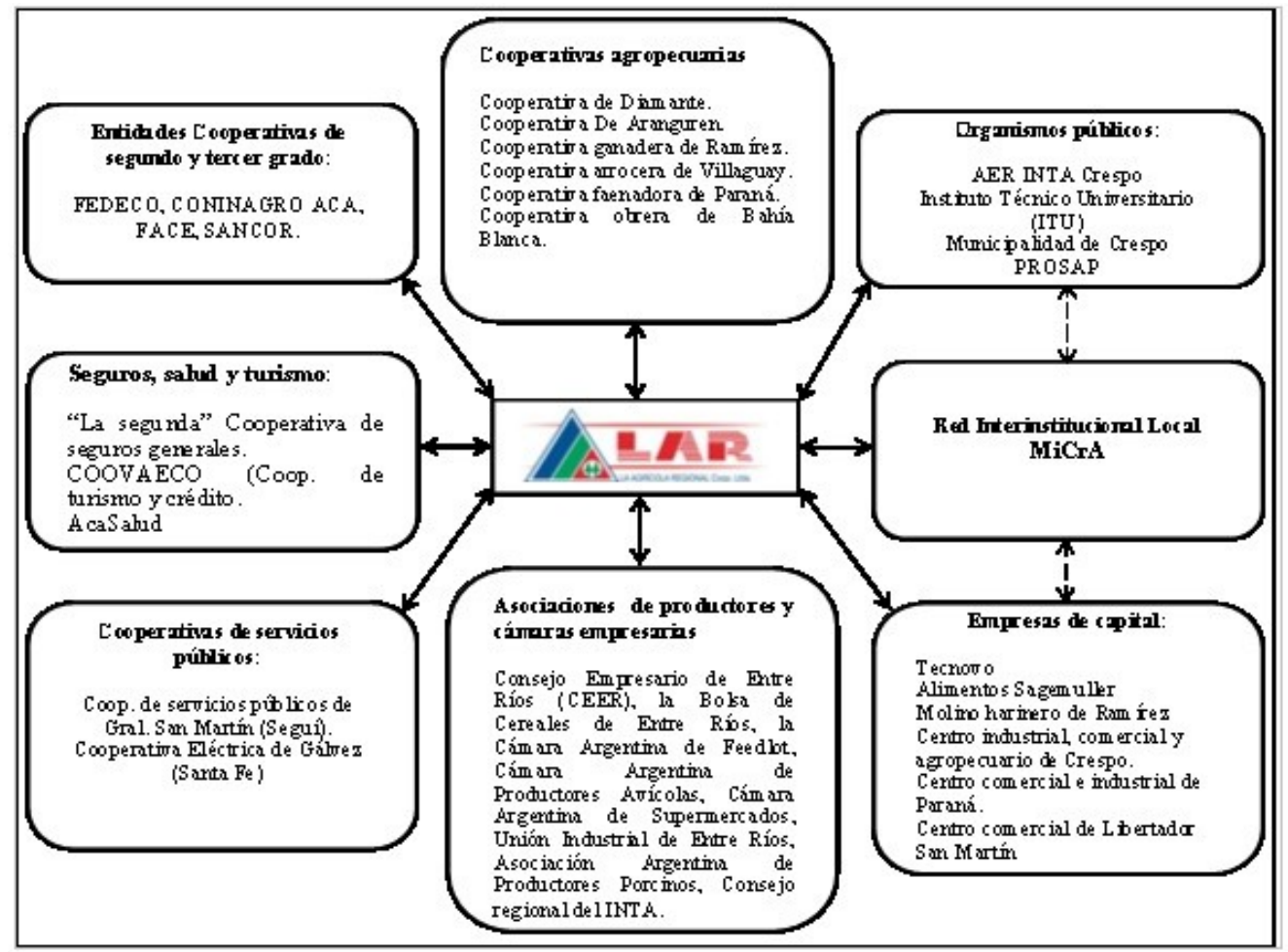

Fuente: Elaboración propia

También resulta importante su relacionamiento con asociaciones de productores, cámaras empresarias y organismos públicos con injerencia en las distintas actividades, como son el Consejo Empresario de Entre Ríos (CEER), la Bolsa de Cereales de Entre Ríos, la Cámara Argentina de Feedlot, Cámara Argentina de Productores Avícolas, Cámara Argentina de Supermercados, Unión Industrial de Entre Ríos, Asociación Argentina de Productores Porcinos, Cámara Argentina de Especias, Consejo del Centro Regional del INTA, entre otras.

Además, se destaca su participación en un proyecto interinstitucional local (Microrregión Crespo y Aldeas Aledañas -MiCrA-), surgido en el seno del Instituto Técnico Universitario (ITU) de la Universidad Autónoma de Entre Ríos, y de la cual también hacen parte el INTA, el Gobierno Municipal, algunas empresas privadas y el Programa de Servicios Agrícolas Provinciales (PROSAP).

Esta capacidad de articulación con actores de distinta índole, exhibe un fuerte anclaje socio-territorial, revistiendo gran importancia para la generación de sinergias positivas y su aporte al desarrollo económico local, siendo relevante para la recirculación de dinero en la ciudad y la región, la generación de empleo directo e indirecto, la mejora en la prestación de servicios para la comunidad, el acceso a energía eléctrica en las zonas rurales y localidades vecinas, la regulación de los precios, beneficios para los comercios minoristas, generación de posibilidades de venta a micro fábricas y comercios locales (oportunidad de comercializar sus productos en los supermercados de LAR), oportunidades de reconversión productiva, generación de economías complementarias y el posicionamiento de Crespo como marca (Pagnone, 2010).

En efecto, LAR ha logrado consolidarse como el mayor referente a nivel local, no sólo desde el punto de vista económico-productivo, sino también como lugar de confluencia para la población rural y de las localidades vecinas que visitan la ciudad: 
La cooperativa es como que ya viene desde mis abuelos, de mi papá, todos viste. Yo lo que veo en Crespo, si la Agrícola no abre, Crespo no existe, es decir, la gente está tan acostumbrada a la cooperativa que es como algo que es parte de uno ya...sí no vas a la cooperativa es como que no fuiste a Crespo (...) Tan importante es para la zona la cooperativa porque viene gente de todos lados (Entrevista a productor hortícola de Aldea Santa Rosa, 2013).

De este modo se podría afirmar que la cooperativa se sostiene en un contexto territorial que le confiere identidad. Al tiempo, las redes que se construyen y la forma en que evolucionan en el tiempo le otorgan determinadas propiedades a dicho contexto, que definen su propia configuración (Shmite, 2009).

Otras acciones que tienen que ver con la generación de capital social al interior del territorio, se relacionan no sólo con la educación y promoción de la actividad cooperativa (Pagnone, 2005; Borche 2011; FEDECOCONINAGRO, 2012), sino también con la formación de personal con capacidad de liderazgo, como factor clave para la continuidad del proyecto en el tiempo.

\section{Consideraciones finales}

Como resultado del análisis se puede observar la forma en que las reconfiguraciones se han llevado a cabo a lo largo de la trayectoria de LAR. Sintéticamente, éstas han consistido en la apertura de nuevas unidades de negocios y sucursales más allá de los límites locales. La conformación de alianzas estratégicas con otras cooperativas y empresas locales, la participación accionaria en empresas de capital (sociedades instrumentales), y un modelo de gestión en el cual la toma de decisiones estratégicas es delegada a una gerencia profesional, han significado un proceso de reproducción ampliada del capital, consolidándola a la vez como una "gran empresa cooperativa" (Lattuada y Renold, 2004).

De hecho, su compleja estructura de negocios y el modelo de gestión instaurado son factores claves que confieren a la organización una gran flexibilidad y capacidad de adaptación frente a las perturbaciones del entorno, logrando hacer frente a situaciones de crisis internas (a nivel país) y externas. En ese sentido, y pese a que esa misma complejidad se ve reflejada en la baja participación de los asociados en las estancias regulares de participación (asambleas), se debe rescatar su capacidad para generar mecanismos a través de los cuales se logre el "acercamiento" a las problemáticas de los asociados. De este modo, pese a ser una organización en constante cambio -donde sin dudas hay una marcada orientación hacía el mercado, aún exhibe (en el discurso y en la práctica) una preocupación por poner en valor los principios fundacionales.

Además en su rol de entidad líder del sistema productivo local, se rescata su importancia en la generación de empleo directo e indirecto, la aparición de actividades conexas, el apoyo a la comunidad, el fomento de la educación cooperativa y en la construcción de iniciativas para la permanencia de la producción familiar en el ámbito rural. Todo esto, sumado a su capacidad de articulación en red con otros actores locales y no locales que trascienden la actividad económico-productiva, se considera de gran relevancia para la conformación de capital social y la concreción de iniciativas de desarrollo local.

Un hallazgo más que interesante en la presente investigación hace referencia al fuerte anclaje socio-territorial de la cooperativa -que atraviesa la mayoría de los encadenamientos productivos locales y la prestación de servicios para sus asociados y la población en general-, una situación no necesariamente encontrada en otras localidades donde se asientan cooperativas agrarias. Esto se da a tal punto que se dificulta concebir el territorio de la microrregión de Crespo sin la presencia de LAR, y la existencia de ésta última separada del ámbito de pertenencia. En ese sentido vale la pena resaltar que la propiedad de la cooperativa por parte de los asociados se convierte en un factor determinante para el enraizamiento de la misma en el espacio local. No obstante, bien vale la pena reflexionar respecto a la medida en que la estrategia de reproducción ampliada que se verifica podría empezar a dar lugar a un proceso de deslocalización, en el cual se prioricen las nuevas redes que se van estableciendo (algunas de ellas con actores extralocales de peso) en detrimento de las ya existentes en el plano 
local.

En términos generales se puede plantear que el análisis realizado constituye un aporte valioso para el avance en el estudio del conocimiento respecto a la forma en que las cooperativas agrícolas pueden lograr su adaptación a los cambios del contexto. Si bien este proceso implica, en la mayoría de los casos, el despliegue de estrategias organizativas e institucionales que propendan a una mayor competitividad en los mercados, también es posible evidenciar la importancia de la conformación de redes de relaciones en distintos niveles. La naturaleza de dichas redes, particularmente ligada a las lógicas que sustentan al movimiento cooperativo, así como a la cultura de negocios, tradiciones y el sistema de valores imperante, puede resultar fundamental para el posicionamiento de las cooperativas en los sistemas productivos locales, no sólo por su importancia económico-productiva, sino también para la construcción identitaria del territorio.

\section{Notas}

1 Para el caso de Entre Ríos se destacan tres leyes fundamentales: $1^{\circ}$ ) La Ley Nacional de Inmigración y Colonización promulgada en 1876, que estimuló la llegada masiva de los inmigrantes europeos; $2^{\circ}$ ) La Ley Provincial de Traza de Villas y Colonias, que concedía tierras gratuitas a las familias colonizadoras, en Entre Ríos, sin discriminación de nacionalidad y 3º La Ley provincial promulgada en 1885 que declaraba de utilidad pública la expropiación de una legua cuadrada a cada costado de la vía del Ferrocarril Entrerriano, para su colonización.

$\underline{2}$ Ese mismo año también surgieron otras dos entidades en la provincia de Buenos Aires, La Previsión, Cooperativa de Seguros Agrícolas, Colonización y Crédito, en Tres Arroyos, y La Liga Agrícola Ganadera, en Junín. En 1905 se fundó en la provincia del Chaco La Cooperativa Agrícola Ganadera Limitada de Colonia Margarita Belén, que se consolidó como la primera dedicada a la explotación algodonera.

$\underline{3}$ Pese a la larga trayectoria y a la persistencia de esta experiencia pionera, no se incluye en la mayoría de trabajos sobre los inicios del cooperativismo argentino. Esto, como se explicará más adelante, puede encontrar explicación en la adopción durante mucho tiempo de la figura de sociedad anónima.

4 Que sería reorganizada en 1933, pasando a conocerse como la Federación Entrerriana de Cooperativas.

$\underline{5}$ Según datos de Obschatko et al. (2011) el 60\% de las cooperativas agropecuarias activas a finales de 2010 se constituyeron a partir del 2001.

$\underline{6}$ Nacido en Stiria (Austria) en el año 1862, emigró a Argentina en 1885 y en 1887 se radicó en la provincia de Entre Ríos. En 1909 asume como gerente del Banco Regional de Crespo, desde donde aprovechó su cercanía con los productores agrícolas para trasladarles su idea de crear una cooperativa en la zona (Wagner, 2011). Todo hace suponer que el nombre La Agrícola Regional fue inspirado por el de la entidad bancaria.

Z Nacido en Moose, Budyandingen, del Gran Ducado de Oldenburg, Alemania, en 1970. Emigró a Argentina en misión comercial en el año 1893. Fue el primer presidente de LAR entre 1910 y 1912.

8 La inmigración comenzó hacía la últimas décadas del siglo XIX y los inicios del siglo XX. Puntualmente los primeros colonos arribaron a la zona en el año 1878, siendo actores fundamentales, junto con grupos menores de inmigrantes italianos, de la configuración socio-productiva local (Wagner, 2011).

9 En 1889 se incorporaron al Código de Comercio los artículos 392, 393 y 394 sobre sociedades cooperativas. Sin embargo, la caracterización era muy pobre y sólo se contemplaba el principio de “un hombre un voto”. Además se aceptaba que las cooperativas se estableciesen bajo cualquiera de las formas societarias mercantiles consagradas.

10 En 1916 se conformó un Seguro Mutuo contra accidentes del personal de trilladoras, que dejó de existir en el año 1922 por falta de interés de los asociados (Wagner, 2011). 
11 La venta fue realizada por la Cooperativa Agrícola La Unión Limitada de general Ramírez, que adquirió el molino en 1973.

$\underline{12}$ El Programa “Soja Solidaria” fue lanzado en 2002 por la Aapresid (Asociación Argentina de Productores de Siembra Directa), en convenio con la fundación Caritas, para dar respuesta a la crisis alimentaria en el país, a través de donaciones del grano, buscando de esa manera su incorporación a la dieta de las familias más carenciadas. No obstante, la iniciativa no duraría mucho dadas las fuertes críticas que enfrentó respecto a los peligros del consumo de soja transgénica.

13 Moneda alternativa emitida por el gobierno de la provincia de Entre Ríos.

$\underline{14}$ En la actualidad nuclea cerca de 70 productores con 11 mil plantas de nuez pecán (Entrevista a Responsable de Área, 2013).

15 En el periodo 2012/2013, el 31.5\% del capital de la cooperativa se concentraba en 50 socios, que a su vez estuvieron involucrados en 13,64\% de la operatoria con asociados (Balance Social, 2013)*-.

16 Su instrumentación persigue distintos objetivos, entre otros, aumentar la recaudación, estabilizar, los precios internos, proteger a las industrias nacientes, modificar la distribución del ingreso. En Argentina particularmente, ha estado ligada a devaluaciones de la moneda doméstica, con el fin de capturar las ganancias extraordinarias originadas en la mejora de los términos de intercambio (Garriga y Rosales, 2008, p. 4).

17 El ejercicio de los titulares dura tres años con la posibilidad de ser reelegidos después de un año de terminado su mandato, renovándose anualmente por terceras partes en cuanto se refiere a los titulares. Los suplentes sólo ofician en el cargo durante un ejercicio y reemplazan por orden de lista a todo miembro titular que renuncie, fallezca, o sea excluido del Consejo de Administración en cuyo caso completará el mandato del miembro reemplazado (Estatuto LAR, creado en 1952 y modificado en 1980).

18 Su designación, y la de su personal de apoyo, es responsabilidad del CDA.

19 Cuando los asociados con derecho a participar en las Asambleas superen los 5000 éstas serán sustituidas por Asambleas de delegados elegidos en Asambleas electorales de distrito en las siguientes condiciones: a) El Consejo de Administración dividirá el padrón de asociados en distritos integrados por mil asociados cada uno, con derecho a elegir diez delegados titulares y diez delegados suplentes por distrito. b) En caso de existir un distrito que no complete el número de mil asociados se procederá a la elección de un delegado titular y un delegado suplente por cada cien asociados que integren el mismo, o fracción no menor de cincuenta.

$\underline{20}$ Los costos fijos se prorratean en base a tres ítems: la facturación, el monto inmovilizado que tuvo cada asociado en base al capital de trabajo y el personal administrativo.

$\underline{21}$ Esta situación dificulta establecer con precisión la superficie productiva total de los asociados a LAR. No obstante, según datos suministrados por la gerencia, durante la temporada 2012-2013 se sembraron 27.000 hectáreas de trigo con semilla suministrada por LAR, lo que nos da una dimensión de la importancia de la actividad agropecuaria dentro de la estructura de negocios de la cooperativa.

\section{Referencias bibliográficas}

Agrícola Regional Cooperativa Limitada Agropecuaria, de Consumo y de Servicios Públicos (2012). Proyecto de Educación Cooperativa “Brisas Del Campo”, de la Agrícola Regional Cooperativa Ltda. [Documento de debate]. Pre Congreso Argentino de las Cooperativas. Rosario, Argentina.

Anello, M. C., Tort, M. I., y Carballo, C. (2012). El cooperativismo agrario y la economía social en las universidades públicas. PRECAC, Facultad de Ciencias Económicas, UBA. 
Barbero, A., Gorenstein, S., y Gutiérrez, R. (2000). "El impacto de los cambios institucionales en el cooperativismo agropecuario pampeano”. Bogotá, Colombia: Pontificia Universidad Javeriana. Seminario Internacional.

Barea, J., Juliá, J.F., y Monzón, J.L. (1999). Grupos empresariales de la economía social en España. Valencia: Ed. CIRIEC.

Barton, D. (1989). Principles. En David Cobia (Ed.) Co-operatives in Agriculture, pp. 21-34. Englewood Cliffs, NJ: Prentice-Hall.

Barton, D. (2000). What is a cooperative? Unpublished paper, Kansas State University, USA.

Basáñes, C. (1999). Debate en el cooperativismo agropecuario. Buenos Aires, Argentina: RBS-USDA.

Basáñes, C. (2004). Nuevas estrategias de las cooperativas agropecuarias. Uruguay: NETICOOP.

Borche, J.P. (2011). Juventudes agrarias cooperativistas en la Cooperativa LA AGRICOLA REGIONAL de Crespo: un estudio de caso enfocado en la educación cooperativa. 1910-2010. V Jornadas uruguayas de historia económica, Montevideo-UDELAR.

Bijman, J., Hendrikse, G., \& Veerman, C. (2000). A marketing cooperative as a system of attributes: A case study of VTN/The Greenery International BV. En J. H. Trienekeus, \& P. J. Zuurbier (Eds.), Chain Management in agribusiness and the food industry (pp. 203-213). Wageningen, The Netherlands: Wageningen Press,

Caldentey, P. (2002). Redes de empresas y cooperativas agrarias de comercialización. Recuperado de: http://www.infoagro.com/agricultura ecologica/cooperativas agrarias.htm. Consulta: 22 enero de 2011.

Caletti, A. (1983). Iniciación, desarrollo y porvenir del cooperativismo. Cuadernos de Cultura Cooperativa No 69. Buenos Aires: INTERCOOP, Editora Cooperativa Ltda.

Chaddad, F.R., y Cook, M.L. (2004). Understanding New Cooperative Models. An Ownership-Control Rights Typology. Review of Agricultural Economics, 26(3), 348-360.

Cook M.L. (1995). The Future of U.S. Agricultural Cooperatives: A Neo-Institutional Aproach. American Journal of Agricultural Economics, 77(5), 1153-1159.

Cook, M.L., \& Iliopoulos, C. (1999). Beginning to Inform the Theory of Cooperative Firm: Emergence of the New Generation Cooperative. The Finish Journal of Business Economics, LTA 4(99), 525-535.

Cook, M.L., \& Iliopoulos, C. (2000). Ill-defined property rights in collective action: the case of US agricultural cooperatives. En C. Ménard, (Ed.), Institutions, Contracts and Organizations: Perspectives from New Institutional Economics (pp. 335-348). Cheltenham: Edward Elgar.

Cracogna, D. (1968). Cooperativismo agrario argentino. Orígenes, estado actual, reestructuración. Buenos Aires: INTERCOOP, Editora Cooperativa Ltda.

De Lisio, C. (2009). Las cooperativas como parte de la economía social, ¿una alternativa para salir de la crisis? [Documento de discusión]. Reunión Especializada de Cooperativas del MERCOSUR (RECM). Uruguay.

Donoso I., Rudzki R., Shadbolt, N., \& Bailey, W. (2003). The Internationalization of Agricultural Co-operatives: Critical Factors in Development. Agribusiness Perspectives Massey University, New Zealand.

Fairbain, B. (2006). Cohesion, adhesion, and identities in co-operatives. Centre for the Study of Co-operatives University of Saskatchewan.

Fulkas, M. (2009). Las pymes argentinas en el escenario post convertibilidad. Políticas públicas, situación y perspectivas. CEPAL. 
Garriga, M., y Rosales, W. (2008). Efectos asignativos, distributivos y fiscales de las retenciones a las exportaciones. Documento de trabajo $n^{\circ}$ 75. FCE UNLP - Unión Industrial del Gran La Plata.

Gorenstein, S., y Peri, G. (1990). Cambios tecnológicos recientes en la Pampa Húmeda Argentina: ¿modifican la dinámica de sus áreas rural - urbana? En Tecnología y reestructuración productiva: impactos y desafíos producción. Buenos Aires: Edit. Grupo Editor latinoamericano.

Gorenstein, S., Barbero, A., y Gutiérrez, R. (2000). El impacto de los cambios institucionales en el cooperativismo agropecuario pampeano. Seminario Internacional. Bogotá, Colombia: Pontificia Universidad Javeriana.

Gorenstein, S., Barbero, A., y Gutiérrez, R. (2001). Nuevas tendencias en el cooperativismo agrario pampeano. Realidad Económica, 181, 108-130.

Gutman, G. (2005). Desarrollo y articulación de PP (pequeños productores) hortícolas en un programa cooperativo: el caso de la Cooperativa LAR, Entre Ríos. En Agricultura de contrato de Pequeños Productores Agropecuarios con Agroindustrias y/o Agrocomercios en Argentina, Experiencias, Lecciones, Lineamientos de Políticas. Buenos Aires: RIMISP.

Holmes, M., Walzer, N., \& Merret, Ch. (2001). New Generation Cooperatives: Case Studies. Illinois Institute for Rural Affairs, Western Illinois University.

King, R.P. (1995). The future of agricultural cooperatives in North America: Discussion. American Journal of Agricultural Economics, 77(5), 1160-1161.

King, R.P. y Ortmann, G.F. (2006). Small-scale farmers in South Africa: Can agricultural cooperatives facilitate access to input and product markets? Department of Applied Economics. College of Agricultural, Food, and Environmental Sciences, University of Minnesota.

Lang, M.G. (1995). The future of agricultural cooperatives in Canada and the United States: Discussion. American Journal of Agricultural Economics, 77(5), 1162-1165.

Lattuada, M. (1995). Globalización, políticas de ajuste, y sector agropecuario en Argentina. Políticas Agrícolas, 1(1).

Lattuada, M. (2006). Acción Colectiva y corporaciones agrarias en la Argentina. Transformaciones institucionales a los fines del siglo XX. Buenos Aires: Universidad Nacional de Quilmes [primera edición].

Lattuada, M. y Renold J.M. (2004). El cooperativismo agrario ante la globalización. Un análisis sociológico de los cambios en su composición, morfología, y discurso institucional. Argentina: Siglo veintiuno editores.

Lattuada M., Nogueira, M.E., Renold, J.M., Y Urcola, M. (2011) El cooperativismo agropecuario argentino en la actualidad. Presentación y análisis de tres casos desde la perspectiva del capital social. Mundo Agrario, 12(23).

Lombardo, P., Ravina, N., Tort, M.I., y Siolotto, R. (2009). Crisis cooperativa y reconversión institucional. Revista de la Facultad de Agronomía, 29(2), 69-79.

López, C.G. (2008). La fuerza del Ideal. Historia del Cooperativismo Agrario Entrerriano y Su Proyección Nacional, 1900-1970. Paraná: Programa Identidad Entrerriana.

Mateo, G. (2011). La Cooperativa Arroceros Villa Elisa, un buen ejemplo de la tradición cooperativista de Entre Ríos (Argentina). Mundo Agrario, 11(22), 00-00.

Nilsson, J. (1998). The Emergence of New Organizational Models for Agricultural Co-operatives. Swedish Journal of Agricultural Research, 28, 39-47.

Nilsson, J. (1999). Co-operatives Organizational Models as Reflections of the Business Enviroments. Swedish University of Agricultural Sciences, Dept of Economics. LTA 4(99), 449-470. 
Nilsson, J. y Germundsson, P. (2000). A New Generation Cooperative of Old Days. An Analysis of Starch Potatoes Cooperative. En M. Kirk, J.W Kramer y R. Steding (Ed.), Genossenschaften und Kooperation Einer Eich Wandelnden Welt (pp. 293-304). Munster.

O'connor, J., y Thompson, G. (2001). International trends in the structure of agricultural cooperatives. Agribusiness Review, 9(9).

Obschatko, E., Basañes, C., y Martini, G. (2011). Las Cooperativas Agropecuarias en la República Argentina. Diagnóstico y propuesta. [1 $1^{\mathrm{a}}$ ed.] Buenos Aires: Ministerio de Agricultura Ganadería y Pesca de la Nación / IICA Argentina.

Olivera, G. (2008). El cooperativismo agropecuario, los chacareros y la renovación del historial rural. el@tina. Revista electrónica de estudios latinoamericanos, 6 (22), 31-48.

Pagnone, D. (2005). La Agrícola Regional Cooperativa Ltda.- Estudio de caso. Un aporte a la construcción de capital social en la comunidad rural y urbana. Premio Nacional de Ética y Responsabilidad Social Empresaria. Instituto Argentino de Responsabilidad Empresarial (IARSE).

Ressel, A. y Silva, N. (2008). Estudio de las cooperativas agrarias en Argentina. En Cooperativas e Integración Regional. La trayectoria de las cooperativas agropecuarias y de ahorro y crédito en el MERCOSUR. Uruguay.

Rodríguez, J.C., Mozas Moral., A., y Bernal, J. E. (2002). Desarrollo local por cooperativas: diversificación productiva en el ámbito rural andaluz. El caso de BEDMAR. REVESCO, Revista de Estudios Cooperativos, 76, 173-189.

Romero F., Álamo, M., Stolze, B., y Alessandrini, M. (2006). Cambios estructurales y transformaciones institucionales en el cooperativismo agropecuario argentino. El caso del sudoeste de la provincia de Buenos Aires. Cultura política y crisis en la provincia de Buenos Aires (1999-2001): las prácticas y los discursos. Secretaria de Ciencia y Tecnología de la Universidad Nacional del Sur (UNS).

Salminis, J. A. (2003). Apuntes sobre la génesis y desarrollo del cooperativismo agropecuario en la República Argentina. Revista de Estudios Cooperativos, 081, 77-99.

Salminis, J. A. (2010). El cooperativismo agropecuario en la República Argentina: notas sobre su origen y evolución. XXII Jornadas de Historia Económica, Río Cuarto, Córdoba.

Shmite, S. M. (2009). Territorio y redes sociales. Una lectura geográfica de los vínculos multiescalares. $2^{\circ}$ Congreso de Geografía de las Universidades Nacionales, La geografía ante la diversidad socio - espacial contemporánea. Universidad Nacional de la Pampa, Santa Rosa.

Stanford, L. y Hogeland, J.A. (2004). Designing Organizations for a Globalized World: Calavao’s Transition from Cooperative to Corporation. American Journal of agricultural Economics, 86(5), 1269-1275.

Verheijen, J.A.G. y Heijbroek, A.M.A. (1994). Cooperatives in changing market conditions. In G.W. Ziggers, J.H. Trienekens, \& P.J.P. Zuurbier (Eds.), Proceedings of the first international conference on chain management in agribusiness and the food industry (pp. 168-174). Wageningen, The Netherlands: Management studies group.

Von Pischke, J.D. y Rouse, J.G. (2004). New strategies for mobilizing capital in agricultural cooperatives. Rome: FAO Corporate Document Repository: Food and Agriculture Organization of the United Nations.

Wagner, O. (Coordinador). (2011). 100 años de trabajo cooperativo. La Agrícola Regional Cooperativa Limitada, agropecuaria, de consumo y de servicios públicos 1910-2010. Santa Fe: Ediciones UNL.

Wilson, W. (1999). Co-operation, some thoughts for the future - A personal view. Journal of the Royal Agricultural Society of England, 160, 99-105. 


\section{Fuentes}

Balance Social de La Agrícola Regional Cooperativa (2012-2013)

Entrevista personal realizada a coordinador del ITU, Crespo (Noviembre de 2013)

Entrevista personal realizada a técnico del ITU, Crespo (Noviembre de 2013)

Entrevista personal realizada a técnico del INTA, Crespo (Noviembre de 2013)

Entrevista personal realizada a encargado de Relaciones Institucionales de La Agrícola Regional Cooperativa (Noviembre de 2013) 